\title{
MAPPING AND SECURITY ANALYSIS OF SENSOR NETWORK FOR ELECTRIC VEHICLES
}

\author{
Szilárd TULOKI ${ }^{1}$, István NAGY² \\ Obuda University, Bánki Donát Faculty of Mechanical and Safety Engineering, Budapest, Hungary \\ ${ }^{1}$ tuszilard@gmail.com \\ ${ }^{2}$ nagy.istvan@bgk.uni-obuda.hu
}

\begin{abstract}
Today's vehicles are becoming more and more complex in order to meet the ever increasing safety and comfort requirements. Most people usually do not consider the car sitting in their driveway to be on the leading edge of new technology. However, for most people, the personal automobile has now become their initial exposure to new intelligent computational technologies such as fuzzy logic, neural networks, adaptive computing, voice recognition and others. This paper explores the sensory-network of electric vehicles and analyses it from a security and reliability perspective.
\end{abstract}

Keywords: sensory network, reliability, on-board control system.

\section{Introduction}

The sensory system of the electric car can be divided into inner sensors (e. g. Crank Position, Wheel speed sensor) and outer sensors (e. g. camera system, GPS).

We focus on exploiting redundancy for sensor networks in the context of spatial interpolation. The network acts as a distributed sampling system, where sensors periodically sample a physical phenomenon of interest, e.g. temperature. For automobiles to continue to improve safety, increase performance, reduce environmental impact, and enhance comfort, the speed, quantity and reliability of data communicated with a car's electronic control unit (ECU) must increase. Advanced control and safety systems - combining multiple sensors, actuators and electronic control units - are beginning to require synchronization and performance beyond the existing standard Controller Area Network (CAN), can provide. The term topology in communication networks refers to the way the devices are linked together in the network. The basic types of network topologies are Bus Topology, Star Topology, Ring Topology, Mesh Topology and Tree Topology. Electric devices for their sensory system mostly use Tree topology \& Mesh topology combined, so in this way fault diagnosis is easy. (Figure 1.) [1, 2]
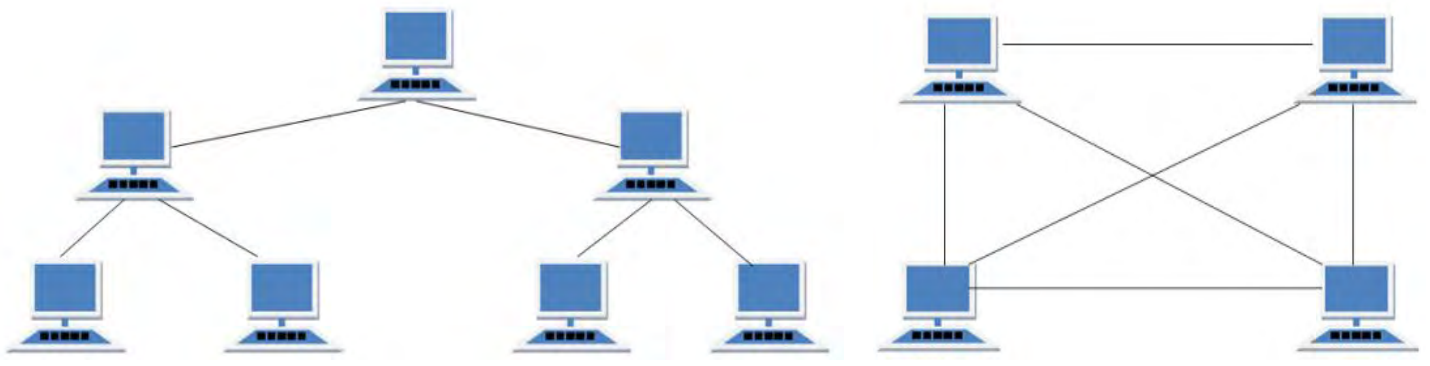

Figure 1. Tree \& Mesh Topology [2] 


\section{Sensor-network topology}

In tree topology, only one route node exists between any two nodes on the network. It is also called hierarchical topology having at least three levels to the hierarchy [2, 3]

Mesh topology is a point to point connection between the nodes or instruction. It allows for continuous connections and reconfiguration around broken and blocked paths by hopping from node to node until a destination is reached [2,3].

\section{The On-board processor possibilities}

\subsection{GPP}

General purpose processors are designed to execute multiple applications and perform multiple tasks. General purpose processors can be quite expensive especially for small devices that are designed to perform special tasks. Also, general purpose processors might lack the high performance that a certain task requires.

\subsection{ASIP}

Therefore, application specific processors have emerged as a solution for high performance and cost effective purposes. Application specific processors have become a part of our lives and can be found almost in every device we use on a daily basis. Devices such as TVs, cell phones, and GPS all have a form of application specific processor. An application specific processor combines high performance, low cost, and low power consumption. [4]

\subsection{ASIC}

Compared to GPP, the ASIC based systems offer better performance and power consumption, but at the cost of flexibility and extensibility. Although it is difficult to use the ASIC for tasks other than what they were designed for, it is possible to use GPP to perform the more generally less demanding tasks in addition to ASIC in the same system [4].

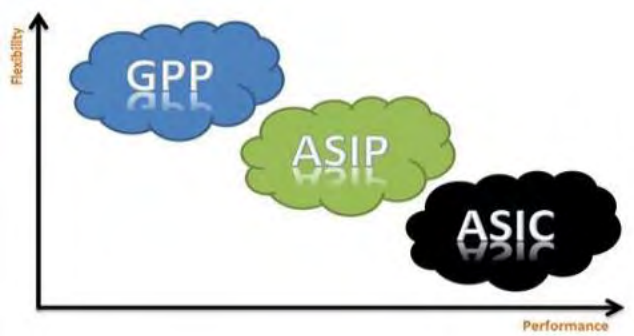

Figure 2. Comparison of the processor's performance vs. flexibility [4]

\subsection{Other technologies}

If we want to further detail the technologies, which were installed, we find different kinds of complex programmable logic devices (CPLD), Field-programmable gate arrays (FPGA), and System on Chips (SoC).

\section{Communication Protocols}

In communication protocols, most cars utilize different kinds of technologies. Most popular are The Universal Asynchronous Receiver Transmitter (UART), Recommended Standard 232 (RS-232), Serial Peripheral Interface Bus (SPI), Inter-Integrated Circuit (I2C), Controlled Area Network (CAN), CANOpen, Local Interconnect Network (LIN), Media Oriented Systems Transport (MOST), FlexRay. Our investigation discovered that the car which we observed used CAN and CANOpen systems.

\subsection{Controller Area Network}

The CAN bus was developed by $\mathrm{BOSCH}$ as a multi-master, message broadcast system that specifies a maximum signalling rate of 1 megabit per second (Mbps). Unlike a traditional network such as USB or Ethernet, CAN does not send large blocks of data point-to-point from node A to node $B$ under the supervision of a central bus master. In a CAN network, many short messages like temperature or RPM are broadcast to the entire network, which provides for data consistency in every node of the system. Originally it was developed for the automotive industry to replace the complex wiring harness with a two-wire bus. The specification calls for high immunity to electrical interference and the ability to self-diagnose

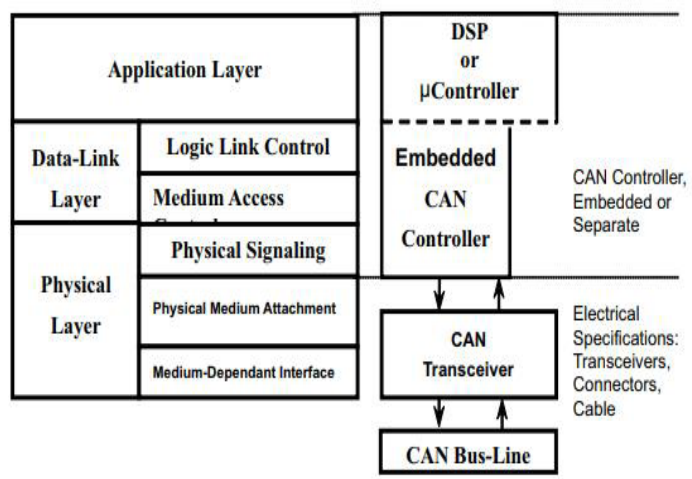

Figure 3. The Layered ISO 11898 Standard Architecture [5] 
and repair data errors. These features have led to CAN's popularity in a variety of industries including building automation, medical, and manufacturing [5]

The application layer establishes the communication link to an upper-level application specific protocol such as the vendor-independent CANopen ${ }^{\mathrm{TM}}$ protocol [5].

\subsection{CANOpen}

Whenever high voltage can damage the end equipment, there is a need for isolation. CANopen unleashes the full power of CAN by allowing direct peer-to-peer data exchange between nodes in an organized and, if necessary deterministic manner. The network management functions specified in CANopen simplify project design, implementation and diagnosis by providing standard mechanisms for network startup and error management. CANopen supports both cyclic and event-driven communication. This makes it possible to reduce the bus load to a minimum but still maintaining extremely short reaction times. High communication performance can be achieved at relatively low baud rates, thus reducing electromagnetic interferences (EMI) and minimizing cable costs. [6]

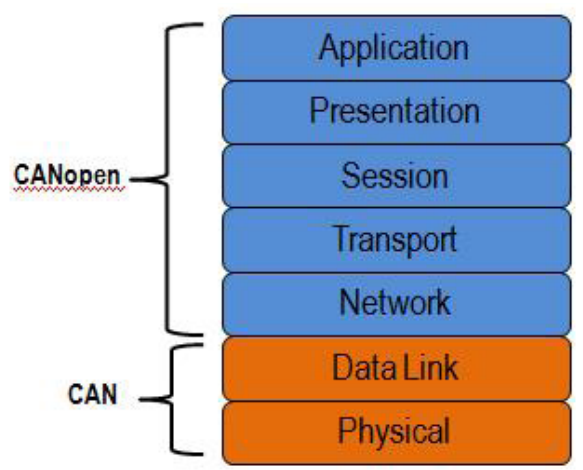

Figure 4. CAN and CANopen in the OSI Model [6]

\section{Vehicle communication system}

Basically, the car communication system can be divided in to 3 main systems

-In-vehicle communication:

The increasing need for bandwidth and the variety of performance, costs and dependability requirements lead to the diversification of the networks used in vehicles. Different networks have different requirements, bandwidth. (i.e. tire pressure sensors).

- Vehicles to vehicles communication:
System designed to transmit basic safety information between vehicles to facilitate warnings to drivers concerning impending crashes.

- Vehicles-satellite communication:

New technology, which provides us with new opportunities in vehicles. Such as emergency responders, vehicle-tracking or up to date traffic track. This technology allows communication in lots of environments, even in the absence of telecom network coverage.

\section{Error propagation analysis in the sensory network of electric vehicles}

In the case of wheel speed sensors, we discovered the following chart, see Figure 5.

\subsection{The AMR sensor analysis}

The anisotropic magnetoresistance (AMR) is used during the convention. Magnetostriction is a property of ferromagnetic materials that causes them to change their shape or dimensions during the process of magnetization. The variation in material's magnetization due to the applied magnetic field changes the magnetostrictive strain until reaching its' saturation value. Since the MR effect cannot measure rotational speed directly, an impulse wheel must deliver the change in magnetic field. These impulse wheels are called encoders. For wheel speed applications there are two types of encoders applicable: magnetized and ferromagnetic. Magnetized encoders have their own magnetic field, while the ferromagnetic encoders can just influence an already existing magnetic field that is generated by a large magnet applied on the sensor.

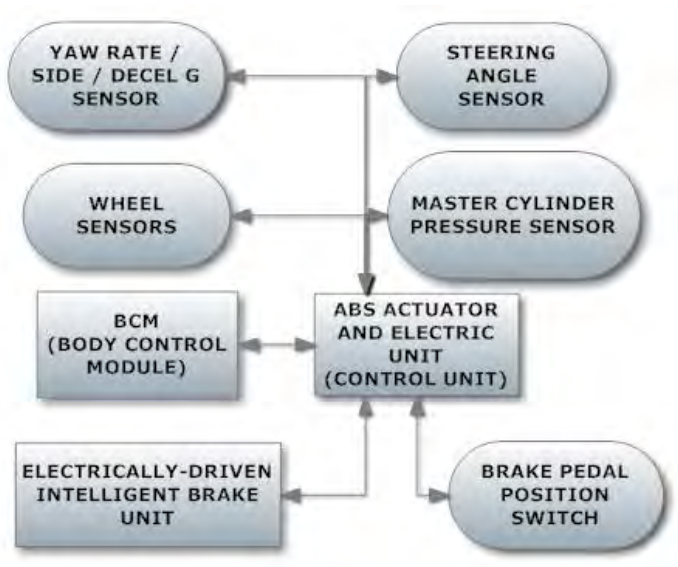

Figure 5. Wheel speed sensor connections 


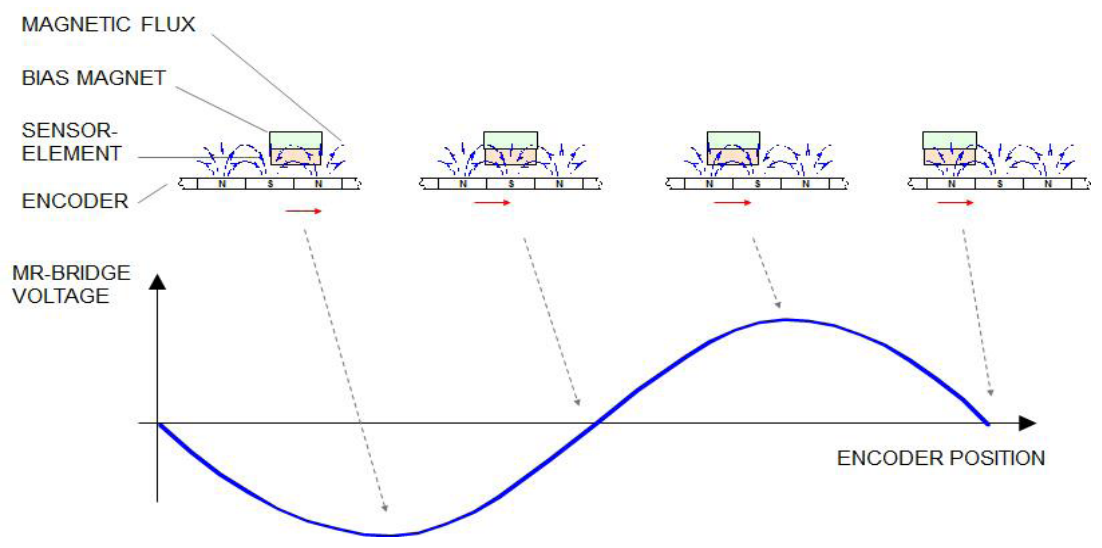

Figure 6. The output signal of AMR bridge with magnetized encoder

\subsection{Conclusion (and possible errors)}

In Figure 5. each of the sensors is able to send an error message, and the control unit is able to send another communication system error message (CAN), which helps in identifying the source of the error. The possible errors can arise from a threshold limit of the analogue sine wave signal, A/D conversion and/or from signal propagation.

\section{Acknowledgement}

This work has been supported by the EFOP 3.6.2-162017-00016 project, which is gratefully acknowledged.

\section{References}

[1] Culler D., Estrin D., Srivastava M. B.: Overview of sensor networks. IEEE Computer 37/8. (2004) 41-49. https://doi.org/10.1109/MC.2004.93
[2] National Instruments: FlexRay Automotive Communication Bus Overview, 2016. http://www.ni.com/white-paper/3352/en/

[3] Nivedita Bisht, Sapna Singh: Analytical study of different network topologies. International Research Journal of Engineering and Technology (IRJET) 2/1. (2015).

[4] Yousef Qasim, Pradyumna Janga, Sharath Kumar, Hani Alesaim: Application specific processors ECE/CS 570 project final report, Oregon State University, 2012.

[5] Corrigan S.: Introduction to the Controller Area Network (CAN), Application report, Texas Instruments, SLOA101B August 2002 Revised May 2016. http:/www.ti.com/lit/an/sloa101b/sloa101b.pdf

[6] National Instruments: The Basics of CANopen. 2013.

http://www.ni.com/white-paper/14162/en/ 